\title{
BMJ Open Implementation of health-focused interventions in vulnerable populations: protocol for a scoping review
}

\author{
Amanda M Midboe, ${ }^{1,2}$ Caroline Gray, ${ }^{1}$ Hannah Cheng (D) , ${ }^{1,2}$ Leonore Okwara, ${ }^{1}$ \\ Randall C Gale ${ }^{1}$
}

To cite: Midboe AM, Gray C, Cheng $\mathrm{H}$, et al. Implementation of health-focused interventions in vulnerable populations: protocol for a scoping review. BMJ Open 2020;10:e036937. doi:10.1136/ bmjopen-2020-036937

- Prepublication history and additional material for this paper are available online. To view these files, please visit the journal online (http://dx.doi. org/10.1136/bmjopen-2020036937).

RCG deceased on 1 September 2018.

Received 10 January 2020 Accepted 05 June 2020

Check for updates

(c) Author(s) (or their employer(s)) 2020. Re-use permitted under CC BY-NC. No commercial re-use. See rights and permissions. Published by BMJ.

${ }^{1}$ Center for Innovation to Implementation (Ci2i), VA Palo Alto Health Care System, Palo Alto, California, USA

2Department of Psychiatry,

Stanford University School of Medicine, Stanford, California, USA

Correspondence to Dr Amanda M Midboe; amanda.midboe@va.gov

\section{ABSTRACT}

Introduction Vulnerable populations face significant challenges in navigating the care continuum, ranging from diagnosis of illness to linkage and retention in healthcare. Understanding how best to move individuals within these vulnerable populations across the care continuum is critical to improving their health. A large body of literature has focused on evaluation of implementation of various health-focused interventions in this population. However, we do not fully understand the unique challenges to implementing healthcare interventions for vulnerable populations. This study aims to examine the literature describing implementation of health service interventions among vulnerable populations to identify how implementations using the Consolidated Framework for Implementation Research are adapted. Findings from this review will be useful to implementation scientists to identify gaps in evidence and for adapting similar interventions in unique settings.

Methods and analysis This study protocol outlines a scoping review of the peer-reviewed and grey literature, using established approaches delineated in Arksey and 0'Malley's scoping review framework and the Preferred Reporting Items for Systematic Reviews and MetaAnalyses extension for Scoping Reviews Checklist. Search strategies will be developed and refined by a medical librarian in collaboration with the research team. Searches will be conducted in electronic databases (CINAHL, Cochrane, PsychINF0, PubMed, Social Services Abstracts, Web of Science, Google and Google Scholar) and limited to studies published between 1 August 2009 and 1 June 2020. Additionally, hand searches will be conducted in three relevant journals-Implementation Science, Systematic Reviews and BMJ Open. English-language studies and reports meeting inclusion criteria will be screened independently by two reviewers and the final list will be abstracted and charted in duplicate.

Ethics and dissemination This is a review of the literature; ethics approval is not indicated. We will disseminate findings from this study in peer-reviewed journals as well as presentations to relevant stakeholders and conferences.

\section{INTRODUCTION}

While anyone can face significant challenges in obtaining necessary and timely healthcare, vulnerable populations can face even greater

\section{Strengths and limitations of this study}

This will be the first scoping review to identify specific examples of how domains and constructs from the Consolidated Framework for Implementation Research have been used to implement and modify interventions and programmes targeted at vulnerable populations.

- Search strategies will be designed for six research databases, a variety of grey literature resources and three peer-reviewed journals covering implementation science.

- Although this study will identify implementation modifications, there will be no formal assessment or comparison of the quality of the studies.

challenges. Vulnerable populations are generally defined as those at risk of harm and neglect due to a lack of resources to help mitigate individual and community-based challenges. ${ }^{1}$ Vulnerability may result from a variety of social, psychological and/or physical challenges accumulating across one's life, which leaves individuals and groups at an increased risk of diminished health outcomes. ${ }^{2} 3$ They may include racial and ethnic minorities, individuals living in impoverished conditions, disabled individuals, elderly persons, individuals living with substance use or other types of mental illness and those who are unstably housed or involved in the criminal justice system. ${ }^{4-6}$ Many of these populations face significant social barriers, notably stigma and economic vulnerability, which further complicate their healthcare challenges. ${ }^{1}$ Vulnerable populations face significant challenges navigating healthcare systems and experience higher rates of morbidity and mortality due to inadequate care. ${ }^{4}$ These populations are also less likely to seek out and use health services, despite their higher needs for these services. Furthermore, the services that vulnerable populations need are often more specialised and require complex coordination among 
medical specialities as well as community-based social services. ${ }^{7}$ These services may be difficult to integrate into existing work practices. Identifying effective ways to implement health services targeted at vulnerable populations is therefore critically needed.

While many healthcare interventions are found to be effective during clinical trials, they do not always make it into practice. ${ }^{8}$ A widely used implementation science framework, the Consolidated Framework for Implementation Research (CFIR), ${ }^{9}$ provides a meta-theoretical framework for examination of factors that may impact implementation effectiveness of healthcare interventions. A key benefit of the CFIR is that it integrates overlapping constructs into an overarching and standardised catalogue of factors relevant for implementation scientists. Since it branches across implementation theories and frameworks, it can be applied across most implementation evaluations and provides a common language to describe relevant implementation factors. The CFIR is organised in five major domains comprised of 39 constructs that are related to implementation-outer setting, inner setting, intervention characteristics, individual characteristics and process. Since its inception in 2009, the application of CFIR to varying stages of implementation has been substantial. ${ }^{10}$ To date, there have been thousands of publications (and counting) across the world that cite the original 2009 article. ${ }^{11}$ Identifying these factors allows practitioners to refine and adjust health services where needed and therefore increases the likelihood of an intervention's uptake.

\section{RATIONALE}

To our knowledge, there has been no comprehensive review of which factors are most relevant to implementation of interventions or practices in healthcare settings serving vulnerable populations. To appropriately tailor implementation strategies for vulnerable populations, the research community must first identify relevant factors that could impact implementation of interventions that have been and may be effective at reaching these target populations as well as identify gaps in the literature that indicate further investigation. For example, the outer setting may be highly relevant in implementation of interventions in unstably housed populations, including the need to rely more on building community partnerships with healthcare systems to achieve initial linkage to care in this population. At the same time, the review may reveal that there may be a gap in knowledge on how best to build those partnerships (eg, policy changes).

\section{OBJECTIVES}

We propose conducting a scoping review that identifies and synthesises findings from CFIR-based research studies of healthcare interventions for vulnerable populations. Because of the popularity and ubiquity of CFIR in implementation research noted previously, we are focusing on CFIR-based studies. These studies share a common language that will allow for easier translation across studies. Our review will be informed by the Behavioural Model for Vulnerable Populations. Our identification of vulnerable groups will rely on subpopulations of adults outlined in the vulnerable domains of the GelbergAndersen model-predisposing, enabling and need. ${ }^{4}$

In addition to helping us identify specific vulnerable groups, the Behavioural Model for Vulnerable Populations, conceptual model, will inform insights generated from this scoping review. ${ }^{3}{ }^{4}$ This model identifies key factors that influence the challenges vulnerable populations face in maintaining and improving their health. The vulnerable populations model is a revision of Andersen's landmark Behavioural Model of Change, ${ }^{4}{ }^{12}$ which describes the interaction between predisposing, enabling and need domains. The vulnerable populations model adapts these three domains to specify how they influence vulnerable populations' healthcare needs and interactions with healthcare systems. In this adapted model, social structure and enabling resources determine 'vulnerability'. Social structure refers to the frequently invisible social arrangements that stratify individuals into different social groups. Social structural characteristics include factors such as residential history, living conditions, mobility, history with the criminal justice system and stigma, characteristics that are highly relevant for the populations our scoping review will target. In their model, homeless persons are identified as a population facing significant social stigma and environmental challenges related to housing instability. These social structural challenges are also experienced among criminal justice-involved populations and those who experience mental illness.

To summarise, we will examine the following vulnerable subpopulations: (1) predisposing-social structure and sexual orientation and other characteristics (eg, homeless, unstably housed, impaired mobility, justice involvement, mental illness, psychological resources (eg, cognitive impairment) and substance use); (2) enabling-individuals receiving public benefits, accessing social services and living in high crime communities and (3) need-individuals with vulnerable health conditions (eg, sexually transmitted infections, maternal care for low-birthweight infants). We will include any study that falls within the subpopulation but have provided some illustrative examples for clarity. Given our goal is not to predict health outcomes; rather, to evaluate relevant factors for implementation of interventions in vulnerable populations, we will not focus on psychosocial constructs such as perceived health (need vulnerable domain).

A limitation is that we will be leaving out some vulnerable subpopulations of interest by not including an exhaustive definition. ${ }^{6}$ However, we believe the trade-off is that the review will provide clearer and less diffuse next steps to implementation scientists interested in intervening with specific vulnerable subpopulations that have welldocumented challenges in engaging in health services 
(eg, individuals who are homeless, living with mental illness). Another limitation of the Behavioural Model for Vulnerable Populations is that it can be challenging to operationalise because of the multiple constructs across domains that are not always defined clearly. ${ }^{13}$ However, we believe this model is the best one available for understanding the factors that play a role in health services use by a wide variety of vulnerable populations. ${ }^{14}{ }^{15}$ To mitigate the impact of this limitation, we will have a priori definitions of all constructs and document during the review process if we discover a need to expand the definition-a process described more fully in the Methods and analysis section.

We will focus on adult subpopulations across these domains for two main reasons: (1) implementation of interventions likely varies between adults and children, making it difficult to draw generalisable conclusions from separate groups and (2) to describe implementation efforts that address vulnerable subpopulations in various stages (risk factors that impact use (predisposing), factors that may impede use (enable) and need for care to address conditions more prevalent in vulnerable populations (need).

Scoping review methodology has been used successfully to map the range of research in specific topic areas and to provide end users with an accessible array of information that may inform their own research. ${ }^{16}{ }^{17}$ Scoping review methodology differs from systematic review methodology in that the aim is to identify areas of consensus and gaps in knowledge and not to assess the rigour of given research findings. Systematic reviews generally require a narrow definition of the scope of the review, ${ }^{18}$ whereas scoping reviews follow a more expansive, exploratory approach. Given the lack of a cohesive synthesis of implementation efforts across vulnerable groups, the scoping review methodology is an important first step for assessing the field more generally. Scoping review methodology will allow us to explore the broad topic of framework-guided implementation in vulnerable populations across study designs in peer-reviewed and grey literature without the constraints of meeting stringent quality filters. These findings may then be used to inform similar studies and interventions aimed at improving health outcomes for vulnerable populations.

\section{METHODS AND ANALYSIS}

Our approach will be based on the steps outlined in Arksey and O'Malley's delineation of scoping review methodology and reporting guideline described in the Preferred Reporting Items for Systematic Reviews and Meta-Analyses extension for Scoping Reviews Checklist. ${ }^{19}{ }^{20}$ We will comprehensively search literature where CFIR $^{9}$ was applied to understand interventions targeted at the domains within the Behavioural Model of Vulnerable Populations. We will limit our search to research studies published between 1 August 2009, when the original CFIR article was first published, and 1 June 2020. In brief, the Arksey and O'Malley's method encompasses the following steps (1) identifying the research question, (2) identifying relevant studies, (3) screening and selecting studies, (4) charting the data, (5) collating, summarising and synthesising the results and finally, (6) consulting with stakeholders. While the last step is unique to their approach, it is a valuable step in the scoping review process. An interdisciplinary study team of experts in quantitative and qualitative methodologies, implementation science and vulnerable populations' healthcare needs will carry out the scoping review.

With assistance from a professional librarian, we will search peer-reviewed and grey literature for Englishlanguage studies. Eligible English-language articles and reports will include those that mention in (1) application of the CFIR as part of implementation or for analysis of an intervention after the fact and (2) implementation of programmes that address the needs of the targeted vulnerable populations.

\section{Stage 1: identifying the research question}

After discussion among the research team and consultation with stakeholders, our team defined the research question as the following: 'What are the CFIR domains and constructs most relevant across the stages of implementation (preimplementation to sustainability) for interventions targeting vulnerable populations, as defined by the Behavioural Model for Vulnerable Populations?' We will rely on a comprehensive set of a priori definitions of our key constructs, working closely with a medical librarian to refine them, and achieving consensus within the study team. As noted previously, we are focusing on three vulnerable domains within the Behavioural Model of Vulnerable Populations.

\section{Stage 2: identifying relevant studies}

Studies for this review will be identified via extensive searches of six bibliographic databases (CINAHL, the Cochrane Library, PsychINFO, PubMed, Social Services Abstracts and Web of Science) as well as Google and Google Scholar. These databases have been used previously in scoping reviews. ${ }^{21}$ We will use a combination of keywords, including specific search terms as displayed in online supplementary appendix 1 for predisposing, enabling and need vulnerable domains and direct reference to CFIR. Studies will be limited to those published between 1 August 2009 and 1 June 2020. Reference lists from papers identified through keyword searches will also be hand-searched for relevant studies. To achieve a comprehensive search, we will also supplement the process by hand-searching all publications in the following three journals that publish implementation science studies and review literature, including Implementation Science, Systematic Reviews and BMJ Open. We will also hand-search Google and cross-check the search results with studies identified in previous steps to ensure that all relevant manuscripts and grey literature are captured. Eligible English-language studies will include those that mention 


\begin{tabular}{|c|c|}
\hline Inclusion & Exclusion \\
\hline $\begin{array}{l}\text { Publications that use } \\
\text { CFIR constructs to inform } \\
\text { interventions for vulnerable } \\
\text { populations }\end{array}$ & $\begin{array}{l}\text { Systematic reviews, } \\
\text { dissertations, theses, } \\
\text { conference presentations, } \\
\text { protocol papers, textbooks }\end{array}$ \\
\hline $\begin{array}{l}\text { All geographical areas but } \\
\text { written in English }\end{array}$ & Language other than English \\
\hline $\begin{array}{l}\text { Published between } 1 \text { August } \\
2009 \text { and } 1 \text { June } 2020\end{array}$ & Published before 2009 \\
\hline Study population is adults & $\begin{array}{l}\text { Study population is } \\
\text { adolescents or children }\end{array}$ \\
\hline $\begin{array}{l}\text { Use of CFIR pre, post or } \\
\text { during an intervention }\end{array}$ & $\begin{array}{l}\text { Use of other/no } \\
\text { implementation frameworks }\end{array}$ \\
\hline $\begin{array}{l}\text { Full-text articles with results } \\
\text { section }\end{array}$ & $\begin{array}{l}\text { Abstract or article missing } \\
\text { description of findings/results }\end{array}$ \\
\hline
\end{tabular}

CFIR, Consolidated Framework for Implementation Research.

(1) application of the CFIR as part of implementation and (2) implementation of programmes that address the needs of vulnerable populations. The searches will be conducted on 15 June 2020.

\section{Stage 3: screening and selecting studies}

We will use Covidence online software to guide the study selection process. General inclusion and exclusion criteria are displayed in table 1 . Some criteria will be determined by the research team 'post hoc' during the screening process as we become increasingly familiar with the topic. Once inclusion and exclusion criteria are determined, such criteria will be applied to screen all studies. Studies from engine searches will be imported to Covidence's online system and will then undergo a two-step screening process. First, members of the team will independently screen the title and abstract of imported studies and generate a list of corresponding inclusion or exclusion criteria to make selections. At least two reviewers must vote on the same study for it to move forward. Second, all members will independently conduct full-text screening to determine whether the study has met the inclusion and exclusion criteria. If a study is voted by at least two reviewers, it will move on to the data extraction phase. Any conflicts will be resolved by discussing the exclusion reasons among the reviewers.

\section{Stage 4: charting the data}

The next stage in the scoping review process involves documenting key elements of selected research studies. Data will be sifted, sorted and charted according to major issues and themes. These processes of sorting and sifting are similar to the systematic coding and analysis of qualitative data, requiring the consistent use of agreed on terms for extracting study elements. For each study that is selected to be part of the scoping review, we will identify the intervention, primary vulnerable groups targeted by the intervention, relevant CFIR domains and subdomains and any effective (or ineffective) implementation strategies targeting a vulnerable population. We will use a data charting form, as described by Arksey and O'Malley. ${ }^{19}$ We will 'test' the data charting form with 5-10 studies for which two reviewers will independently extract information from identified studies. This will increase the validity of research findings and later synthesis. We will make refinements to the data charting form as needed. Table 2 is an illustration of how we plan to extract and document findings from the scoping review.

\section{Stage 5: collating, summarising and synthesising results}

This stage of the scoping review involves taking previously demarcated findings and collating them to create larger thematic findings. This will entail grouping together similar findings, summarising those findings and then identifying areas where findings coalesce as well as noteworthy gaps in the literature. We will look for similarities and differences among each vulnerable population and subsequently look across groups to identify common themes.

\section{Stage 6: consulting with stakeholders}

Consistent with recommendations from others who have engaged in scoping reviews, ${ }^{319}$ we will convene a team of stakeholders to assess findings. Stakeholders will consist of experts in CFIR, experts in clinical interventions that target the vulnerable populations we will examine in our scoping review, clinicians and patients. They will be asked to provide insights not readily apparent in the literature. As experts in CFIR, these stakeholder consultants will be engaged throughout the scoping review and can provide useful guidance on unearthing CFIR-guided implementations among vulnerable populations. To facilitate knowledge translation and dissemination in real-world settings, our research team will also consult patients and providers

Table 2 Example of programme implementation using the CFIR ${ }^{9}$

\begin{tabular}{lllll}
\hline $\begin{array}{l}\text { Programme or practice } \\
\text { change implemented }\end{array}$ & $\begin{array}{l}\text { Vulnerable } \\
\text { population }\end{array}$ & CFIR domain & CFIR construct & Findings \\
\hline $\begin{array}{l}\text { Supportive housing } \\
\text { programme }\end{array}$ & $\begin{array}{l}\text { Unstably housed, } \\
\text { mental illness } \\
\text { disorders }\end{array}$ & Inner setting & $\begin{array}{l}\text { Networks and } \\
\text { communications }\end{array}$ & $\begin{array}{l}\text { Need for enhanced communication and } \\
\text { networking with local services dedicated } \\
\text { to addressing housing needs }\end{array}$ \\
\hline
\end{tabular}

CFIR, Consolidated Framework for Implementation Research. 
through our facility's patient and family-centred council. The patient and family council will also provide assistance with optimising the presentation of findings to other key health system stakeholders. ${ }^{21}$ We will plan to meet with the team of stakeholders after all data have been collected and then again once data have been synthesised.

\section{Patient and public involvement}

No patient was involved during the design and development of this scoping review protocol. However, patients will be consulted during 'Stage 6: consulting with stakeholders' of the scoping review process to provide insights into supplement our literature review findings.

\section{ETHICS AND DISSEMINATION}

The primary aim of this scoping review is to inform research-guided interventions that target vulnerable populations, a research topic has not yet been fully explored. Because interventions for vulnerable populations often require additional coordination and outreach, information that can ease this process is greatly needed. Since we are not collecting primary data in this review of the literature, ethics approval is not indicated. The synthesis achieved through this scoping review will facilitate early identification of factors most likely to impact implementation of interventions targeting highly vulnerable populations. Identifying strategies that improve uptake of these interventions will improve health outcomes for vulnerable populations and may ultimately help reduce disparities often experienced among these populations. We will disseminate findings from this study in peer-reviewed journals as well as presentations to relevant stakeholders and conferences.

Acknowledgements Christopher Stave, Graduate/Clinical Education Librarian at Lane Medical Library, Stanford University, provided technical training on identifying search strategies and terms. Laura Damschroder, Research Investigator at Ann Arbor VA HSR\&D/Center for Clinical Management Research, contributed to development and refinement of the search strategies surrounding the CFIR framework.

Contributors AMM conceived of the idea. AMM and RCG developed the research question and study methods. AMM, RCG, LO, CG and HC contributed meaningfully to the drafting, editing and approval of the final manuscript.

Funding This material is based on work supported by Department of Veterans Affairs, Bridge QUERI Program, QUE 15-284.

Competing interests None declared.

Patient and public involvement Patients and/or the public were not involved in the design, or conduct, or reporting, or dissemination plans of this research.

Patient consent for publication Not required.

Provenance and peer review Not commissioned; externally peer reviewed.

Open access This is an open access article distributed in accordance with the Creative Commons Attribution Non Commercial (CC BY-NC 4.0) license, which permits others to distribute, remix, adapt, build upon this work non-commercially, and license their derivative works on different terms, provided the original work is properly cited, appropriate credit is given, any changes made indicated, and the use is non-commercial. See: http://creativecommons.org/licenses/by-nc/4.0/.

\section{ORCID iD}

Hannah Cheng http://orcid.org/0000-0001-8144-5340

\section{REFERENCES}

1 Mechanic D, Tanner J. Vulnerable people, groups, and populations: societal view. Health Aff 2007;26:1220-30.

2 Flaskerud $\mathrm{JH}$, Winslow BJ. Conceptualizing vulnerable populations health-related research. Nurs Res 1998;47:69-78.

3 Oliver S, Peersman G. Making research more useful: integrating different perspectives and different methods. In: Using research for effective health promotion, 2001.

4 Gelberg L, Andersen RM, Leake BD. The behavioral model for vulnerable populations: application to medical care use and outcomes for homeless people. Health Serv Res 2000;34:1273-302.

5 Grabovschi C, Loignon C, Fortin M. Mapping the concept of vulnerability related to health care disparities: a scoping review. BMC Health Serv Res 2013;13:94.

6 O'Neill J, Tabish H, Welch V, et al. Applying an equity lens to interventions: using PROGRESS ensures consideration of socially stratifying factors to illuminate inequities in health. J Clin Epidemiol 2014;67:56-64

7 Baum FE, Bégin M, Houweling TAJ, et al. Changes not for the fainthearted: reorienting health care systems toward health equity through action on the social determinants of health. Am J Public Health 2009;99:1967-74.

8 Stetler CB, Mittman BS, Francis J. Overview of the Va quality enhancement research Initiative (QUERI) and QUERI theme articles: QUERI series. Implement Sci 2008;3:8.

9 Damschroder LJ, Aron DC, Keith RE, et al. Fostering implementation of health services research findings into practice: a consolidated framework for advancing implementation science. Implement Sci 2009;4:50.

10 Kirk MA, Kelley C, Yankey N, et al. A systematic review of the use of the consolidated framework for implementation research. Implement Sci 2016;11:72.

11 CFIR Research Team-Center for Clinical Management Research. Articles \& highlights: featured CFIR articles. Consol Framew Implement Res 2016.

12 Andersen R. A behavioral model of families' use of health services. Chicago: Center for Health Administration Studies, 1968. https:// www.cabdirect.org/cabdirect/abstract/19702701913

13 Babitsch B, Gohl D, von Lengerke T. Re-revisiting Andersen's Behavioral Model of Health Services Use: a systematic review of studies from 1998-2011. Psycho-Soc Med 2012;9:Doc11.

14 Small LFF. Use of mental health services among people with co-occurring disorders and other mental health co-morbidities: employing the behavioral model of vulnerable populations. Ment Health Subst Use 2010;3:81-93.

15 Stein JA, Andersen R, Gelberg L. Applying the Gelberg-Andersen behavioral model for vulnerable populations to health services utilization in homeless women. J Health Psychol 2007;12:791-804.

16 Levac D, Colquhoun H, O'Brien KK. Scoping studies: advancing the methodology. Implement Sci 2010;5:69.

17 Grant MJ, Booth A. A typology of reviews: an analysis of 14 review types and associated methodologies. Health Info Libr J 2009;26:91-108.

18 Armstrong R, Hall BJ, Doyle J, et al. Cochrane Update. "Scoping the scope" of a cochrane review. J Public Health Oxf Engl 2011;33:147-50.

19 Arksey H, O'Malley L. Scoping studies: towards a methodological framework. Int J Soc Res Methodol 2005;8:19-32.

20 Tricco AC, Lillie E, Zarin W, et al. PRISMA extension for scoping reviews (PRISMA-ScR): checklist and explanation. Ann Intern Med 2018;169:467.

21 Jolley RJ, Lorenzetti DL, Manalili K, et al. Protocol for a scoping review study to identify and classify patient-centred quality indicators. BMJ Open 2017;7:e013632.

22 Gilmer TP, Katz ML, Stefancic A, et al. Variation in the implementation of California's full service partnerships for persons with serious mental illness. Health Serv Res 2013;48:2245-67. 